\section{CANCER GENETICS}

\section{Women only}

Women-only nights can be fun, but other events that occur exclusively to women are not so great. Why, for example, should only women who inherit a mutation in the BRCA1 tumour suppressor be prone to breast cancer? Shridar Ganesan et al. have shown, in the November 1 issue of Cell, that BRCA1 might be involved in X inactivation. Perhaps this could explain this female-specific phenomenon.

BRCA1 was known to localize to the unpaired $\mathrm{X}$ chromosome in pachytene spermatocytes, and Ganesan et al. confirmed this by showing that BRCA1 colocalized with a component of the XY body. The XY body shows similarities to the inactive $\mathrm{X}(\mathrm{Xi})$ chromosome in female somatic cells - both are heterochromatic, silenced and are coated with the non-coding XIST RNA. So, does Xi also localize BRCA1?

Immunofluorescence of BRCA1 and fluorescent in situ hybridization (FISH) of XIST, carried out on female human cell lines, revealed that BRCA1 and XIST could colocalize to a nuclear structure - FISH of an $\mathrm{X}$-chromosome probe confirmed that this was one of the $\mathrm{X}$ chromosomes. Chromatin immunoprecipitation analysis - using antibodies to BRCA1 or its binding partner BARD1 - followed by reversetranscriptase PCR (RT-PCR) of XIST confirmed this interaction.

The next step was to investigate what happened in BRCA1-deficient cells. Frozen sections from sporadic breast and ovarian cancers were examined, and although the majority had nuclear BRCA1 and focal XIST (as opposed to diffuse) staining, those from $B R C A 1$-deficient women did not. The HCC1937 human breast cancer cell line - which contains a germline mutation in one BRCA1 allele and has lost the wild-type allele - also lacked focal XIST staining. This could be restored by ectopic expression of wild-type $B R C A 1$, but not of cancerassociated BRCA1 mutants. Similarly, RNAi of BRCA1 in wild-type cells decreased focal staining of XIST.

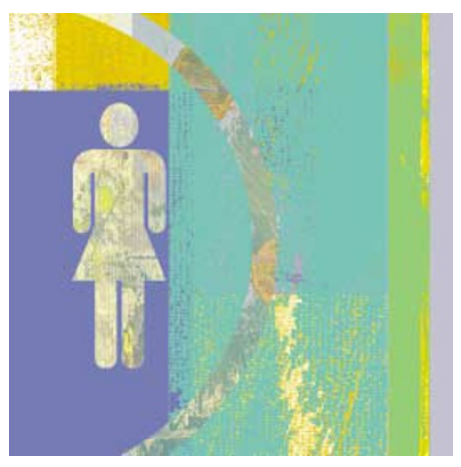

So how does BRCA1 regulate XIST - through its localization, synthesis or stability? The authors carried out RT-PCR of XIST in HCC1937 cells that were transfected with either a vector control or with wild-type $B R C A 1$, to distinguish between these alternatives. The levels of XIST RNA were equivalent in both transfected lines, indicating that BRCA1 can influence XIST localization, but not its synthesis or stability.

As XIST is required for X-chromosome inactivation, Ganesan et al. investigated whether loss of $B R C A 1$ influences the pattern of histone $\mathrm{H} 3$ methylation on lysine 9 ( $\mathrm{H} 3 \mathrm{mK} 9)$, which is associated with transcriptional silencing. In female cells, there is a large amount of anti-H3mK9 antibody staining on $\mathrm{Xi}$, but this is absent from HCC1937 cells. Similarly, $\mathrm{H} 3 \mathrm{mK} 9$ immunofluorescence analysis on frozen sections of sporadic and BRCA1-deficient breast cancers indicates that BRCA1 is required for focal staining of $\mathrm{H} 3 \mathrm{mK} 9$, and hence gene silencing.

But is loss of BRCA1 expression sufficient to reactivate previously silenced genes? This was tested in a female mouse cell line in which one $\mathrm{X}$ chromosome carried a non-functional copy of Xist and the other, inactivated, $\mathrm{X}$ chromosome carried a silenced copy that was tagged with GFP. RNAi of Brcal resulted in the reactivation of Xist-GFP in a subset of these cells.

The loss of BRCA1 in women might therefore reactivate genes that are normally silent on the Xi. The upregulation of a set of Xchromosomal genes in BRCA1-deficient ovarian cancers lends support to the importance of this phenomenon in promoting tumorigenesis, but the establishment of a firm link remains a future goal.

Emma Greenwood, Senior Editor, Nature Reviews Cancer

(2) References and links ORIGINAL RESEARCH PAPER Ganesan, S. et al. BRCA1 supports XIST RNA concentration on the inactive X chromosome. Cell 111, 393-405 (2002) WEB SITE

WEB SITE edu/dms/bbs/fac/livingston.html

\section{IN BRIEF}

\section{FUNCTIONAL GENOMICS}

Genome-wide DNA replication profile for Drosophila melanogaster: a link between transcription and replication timing.

Schübeler, D. et al. Nature Genet. 32, 438-442 (2002)

It has been speculated that a link exists between the time at which a genomic region is replicated in $S$ phase and its transcriptional activity, predominantly because early replication might allow DNA to be packaged into an 'open' chromatin conformation. A microarray-based approach for associating replication timing with gene expression found just such a correlation in Drosophila. This property might distinguish metazoans from lower eukaryotes, such as budding yeast, in which no correlation was found.

\section{DEVELOPMENTAL BIOLOGY}

Different regulation of T-box genes Tbx4 and Tbx5 during limb development and limb regulation.

Khan, P. et al. Dev. Biol. 250, 383-392 (2002)

The limb identity gene Tbx5 promotes limb initiation by interacting with Wnt2b and Fgf10.

Ng, J. K. et al. Development 129, 5161-5170 (2002)

T-box genes $T b x 4$ and $T b x 5$ have been implicated in hindlimb and forelimb development, respectively. Khan et al. cloned Tbx4 and $T b \times 5 \mathrm{cDNAs}$ from newt and showed that, unlike in higher vertebrates, both genes are expressed in the fore- and the hindlimbs. Interestingly, the authors found that, during limb regeneration, their expression is reminiscent of higher vertebrates' $-T b \times 5$ is preferentially upregulated in the forelimbs and $T b x 4$ in the hindlimb - indicating that regeneration is not simply a reiteration of development. An additional role for Tbx5 is revealed by Ng et al., who used gainof-function experiments in chick and zebrafish mutants and morpholino-based knock-down to show that $T b \times 5$, together with $W n t 2 a$, is also necessary and sufficient for limb outgrowth. The authors also show that Tbx5 lies downstream of WNT and that it acts in a feedback loop with Fgflo.

\section{TECHNOLOGY}

\section{Production of maternal-zygotic mutant zebrafish by} germ-line replacement

Ciruna, B. et al. Proc. Natl Acad. Sci. USA 27 October 2002 (10.1073/pnas.222459999)

In zebrafish, and other organisms, maternally contributed RNAs and proteins can mask the effects of zygotic mutations. To overcome this, Ciruna et al. generated sterile fish by ablating primordial germ cells (PGCs) using morpholino oligos against the PGC transcript, dead end. PGCs from homozygous mutant donors were then transplanted into the sterile fish, where they repopulated the gonad and gave rise to maternal-zygotic mutants. This technique could be used to create large clutches of purely mutant embryos. 